\title{
NEW UNIVERSAL FILTER USING TWO CURRENT-FEEDBACK AMPLIFIERS
}

\author{
MUHAMMAD TAHER ABUELMA'ATTI* and \\ HUSAIN ABDULLAH AL-ZAHER \\ King Fahd University of Petroleum and Minerals, Box 203 Dhahran 31261 \\ Saudi Arabia
}

(Received 23 June 1996; In final form 30 August 1996)

\begin{abstract}
A new universal voltage-mode second-order filter circuit is presented. The circuit has three inputs and one low-impedance output and can realize all the standard filter functions; lowpass, highpass, bandpass, notch, and allpass, without changing the passive elements. The proposed circuit uses only five passive components and enjoys independent control of the natural frequency and the bandwidth, orthogonal control of the natural frequency and the quality factor, as well as low active and passive sensitivities.
\end{abstract}

\section{INTRODUCTION}

At present there is a growing interest in designing active filters using current-feedback amplifiers (CFAs) [1-7]. This is attributed to the extended operating bandwidths and relatively large values of slew rates compared to the conventional realizations using the voltage-feedback amplifiers. In [2], a voltage-mode highpass and bandpass filter using two CFAs is proposed and in [3] a voltage-mode bandpass and highpass/lowpass filters using one CFA are proposed. Both circuits are with one input and two outputs. In [4], a voltage-mode highpass/bandpass/lowpass filter using one CFA, but with different passive components, is proposed. In [1], a voltagemode notch, lowpass, and bandpass filter using three CFAs and five passive components; namely, three floating resistors and two grounded ca-

\footnotetext{
*Corresponding author.
} 
pacitors, is proposed. The circuit has one input and three low-impedance outputs. In [5] and [7], two universal filters using two CFAs and six passive elements each; namely four resistors and two capacitors, are presented. Each circuit has three inputs and one low impedance output and uses the same passive components for all realizations. In [6], a high input impedance filter is presented. The circuit can be configured to realize either a bandpass or a lowpass or a highpass filter function. The circuit uses five passive elements; namely, three (or two) capacitors and two (or three) resistors depending on the required filter function. The three filter functions cannot be realized without changing the locations of the passive components used.

The major intention of this paper is to present a new universal filter using CFAs. The proposed circuit enjoys the following advantages:

a. it uses two CFAs.

b. it uses five passive elements only; namely, two capacitors and three resistors.

c. it can realize all the filter functions; namely, lowpass, highpass, bandpass, notch, and allpass, without changing the topology or the number of resistors and capacitors used.

d. it enjoys independent tuning of the natural frequency, $\omega_{o}$, and the bandwidth, $\omega_{d} / Q_{o}$.

e. it enjoys orthogonal tuning of the natural frequency, $\omega_{o}$, and the quality factor, $Q_{o}$.

f. it has one low-impedance output and can, therefore, be easily cascaded to produce higher order filters.

\section{PROPOSED CIRCUIT}

The proposed circuit is shown in Fig. 1. Assuming ideal current-feedback amplifiers with $i_{z}=i_{x}, i_{y}=0, v_{x}=v_{y}$, and $v_{o}=v_{z}$ routine analysis shows that the transfer function of the circuit of Fig. 1 can be expressed as

$$
V_{o}=\frac{s^{2} C_{2} C_{3} V_{3}-s G_{5} C_{2} V_{2}+G_{1} G_{5} V_{1}}{s^{2} C_{2} C_{3}+s G_{4} C_{2}+G_{1} G_{5}}
$$




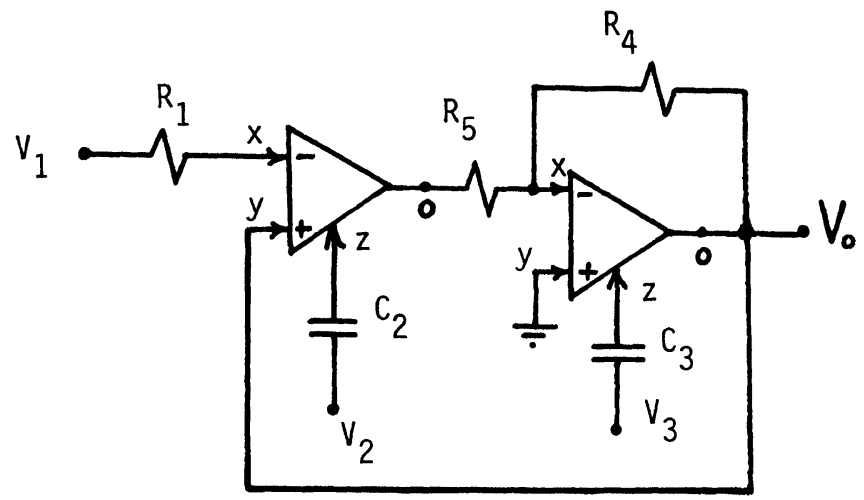

FIGURE 1 Proposed universal filter.

From (1), one can see that:

(i) a second-order lowpass filter can be obtained with $V_{d} V_{1}$ if $V_{2}=V_{3}$ $=0$ (grounded)

(ii) a second-order bandpass filter can be obtained with $V_{d} / V_{2}$ if $V_{1}=V_{3}$ $=0$ (grounded)

(iii) a second-order highpass filter can be obtained with $V_{d} / V_{3}$ if $V_{1}=V_{2}$ $=0$ (grounded)

(iv) a second-order notch filter can be obtained with $V_{\delta} / V_{\text {input }}$ if $V_{2}=0$ (grounded) and $V_{1}=V_{3}=V_{\text {input }}$

(v) a second-order allpass filter can be obtained with $V_{d} V_{\text {input }}$ if $V_{1}=$ $V_{2}=V_{3}=V_{\text {input }}$ and $R_{4}=R_{5}$.

The proposed circuit is attractive for realizing the lowpass filter function, because the two capacitors are grounded. The circuit requires the minimum number of active and passive components with no requirement for match components except for the allpass filter realization. Moreover, the impedance of the single output is very small, so the proposed circuit can be easily cascaded to produce higher order filter realizations. From (1), the parameters $\omega_{o}^{2}$ and $\omega_{d} / Q_{o}$ can be expressed as

$$
\omega_{o}^{2}=1 / C_{2} C_{3} R_{1} R_{5}
$$

and 


$$
\frac{\omega_{o}}{Q_{o}}=1 / C_{3} R_{4}
$$

From (2) and (3), one can see that the parameter $\omega_{o}$ can be adjusted by tuning $C_{2}$ and/or $R_{1}$ and/or $R_{5}$ without disturbing the parameter $\omega_{d} / Q_{o}$. Also, the parameter $\omega_{d} / Q_{o}$ can be adjusted by tuning $R_{4}$ without disturbing the parameter $\omega_{d} / Q_{o}$. Thus, the parameters $\omega_{o}$ and $\omega_{d} / Q_{o}$ are independently adjustable. From (2) and (3), $Q_{o}$ can be expressed as

$$
Q_{o}=R_{4}\left(C_{3} / C_{2} R_{1} R_{5}\right)^{1 / 2}
$$

From (2) and (4), one can easily see that the parameters $\omega_{o}$ and $Q_{o}$ can be orthogonally adjusted.

Taking into consideration the nonidealities of current-feedback amplifiers, namely, $i_{z}=\alpha i_{x}, \alpha=1-\psi_{1},\left|\psi_{1}\right| \ll 1$, denotes the input voltage tracking error, $v_{x}=\beta v_{y}, \beta=1-\psi_{2},\left|\psi_{2}\right| \ll 1$ denotes the input voltage tracking error, and $v_{o}=\gamma v_{z}, \gamma=1-\psi_{3},\left|\psi_{3}\right| \ll 1$ denotes the output voltage tracking error, the transfer function (1) becomes

$$
V_{o}=\frac{\left(\alpha_{1} \alpha_{2} \gamma_{1}\right) G_{1} G_{5} V_{1}-\left(\alpha_{2} \gamma_{1}\right) s C_{2} G_{5} V_{2}+\alpha_{2} \gamma_{2} s^{2} C_{2} C_{3} V_{3}}{s^{2} C_{2} C_{3}+\alpha_{2} \gamma_{2} s C_{2} G_{4}+\left(\alpha_{1} \alpha_{2} \beta_{1} \gamma_{1} \gamma_{2}\right) G_{1} G_{5}}
$$

From (5), the parameters $\omega_{o}^{2}$ and $\omega_{d} / Q_{o}$ can be expressed as

$$
\omega_{o}^{2}=\alpha_{1} \alpha_{2} \beta_{1} \gamma_{1} \gamma_{2} / C_{2} C_{3} R_{1} R_{5}
$$

and

$$
\frac{\omega_{o}}{Q_{o}}=\alpha_{2} \gamma_{2} / C_{3} R_{4}
$$

It is easy to show that the passive and active sensitivities of the parameters $\omega_{o}$ and $\omega_{d} / Q_{o}$ can be expressed as

$$
S_{\alpha_{1}, \alpha_{2}, \beta_{1}, \gamma_{1}, \gamma_{2}}^{\omega_{o}}=-S_{R_{1}, R_{5}, C_{2}, C_{3}}^{\omega_{o}}=1 / 2, S_{C_{3}, R_{4}}^{\omega_{o} / Q_{o}}=-S_{\alpha_{2}, \gamma_{2}}^{\omega_{o} / Q_{o}}=-1
$$

and 


$$
S_{\beta_{2}}^{\omega_{o}}=S_{\alpha_{1}, \beta_{1}, \beta_{2}, \gamma_{1}}^{\omega_{d} / Q_{o}}=0
$$

Thus, all the active and passive sensitivities are no more than unity.

The proposed circuit was tested experimentally and the results obtained from lowpass, highpass, bandpass, and notch filter realizations using the AD844 current-feedback amplifier are shown in Fig. 2. The experimental results are in good agreement with the theory presented.

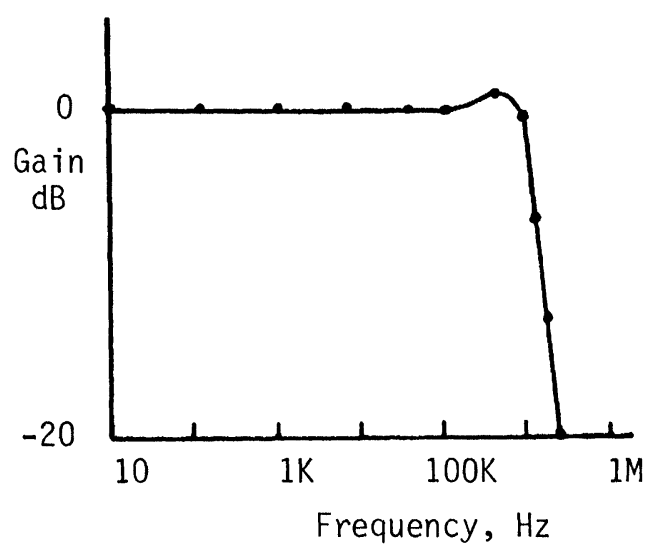

a lowpass characteristic obtained with $R_{4}=R_{5}=2 \mathrm{~K}, R_{1}=1 \mathrm{~K}, C_{2}=C_{3}=100 \mathrm{pF}$.

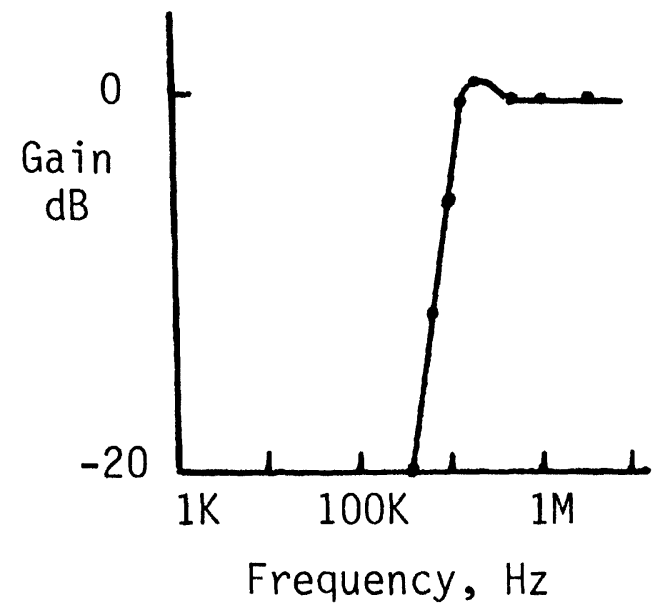

b highpass characteristic obtained with $R_{4}=R_{5}=2 \mathrm{~K}, R_{1}=1 \mathrm{~K}, C_{2}=C_{3}=100 \mathrm{pF}$. FIGURE 2 Measured characteristics of the universal filter of Fig. 1 (continued). 


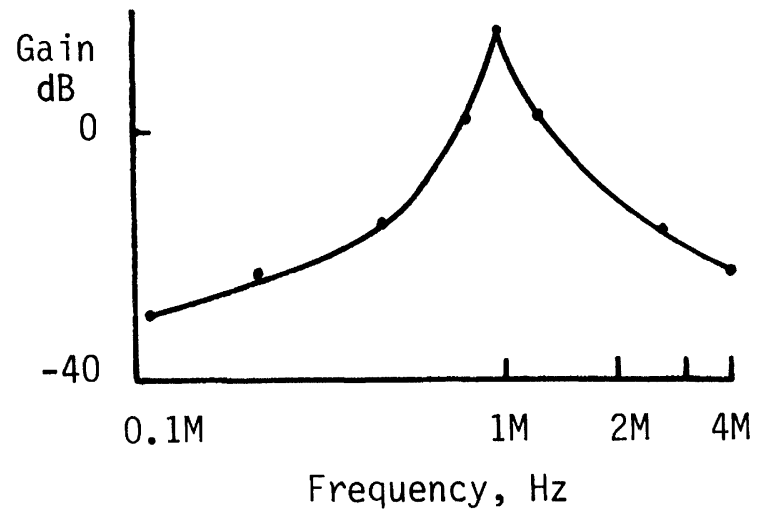

c bandpass characteristic obtained with $R_{1}=1 K, R_{4}=10 K, R_{5}=2 K, C_{2}=C_{3}=100 p F$.

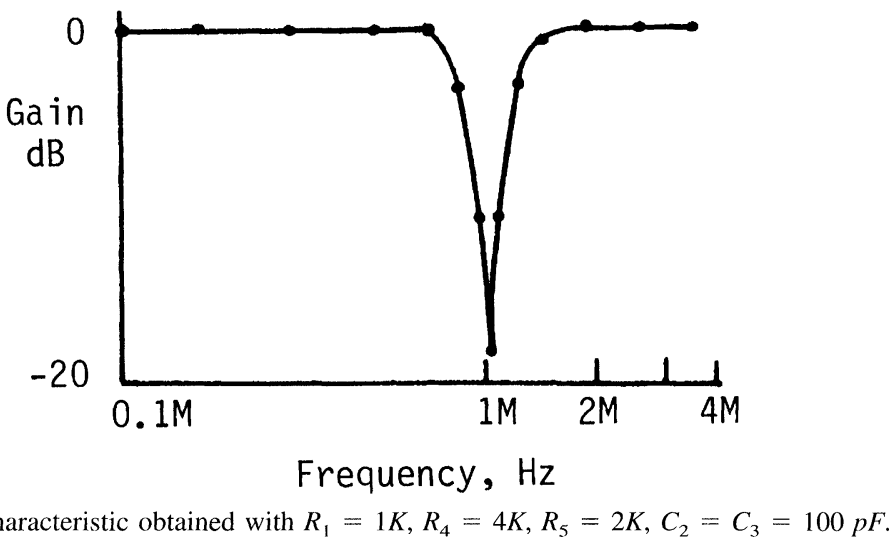

FIGURE 2 (continued).

\section{CONCLUSION}

A new minimum component universal filter using two current-feedback amplifiers, two capacitors, and three resistors has been presented. The new circuit uses less number of passive components and enjoys all the following attractive features: independent control of the natural frequency and the bandwidth, orthogonal control of the natural frequency and the quality factor, low active and passive sensitivities, low output impedance, and the same passive components are used to realize all the standard filter functions; lowpass, highpass, bandpass, notch, and allpass with no component matching requirement except for the allpass realization. 


\section{References}

[1] Chang, C.-M., Hwang, C.-S. and Tu, S.-H. (1994). Voltage-mode notch, lowpass and bandpass filter using current-feedback amplifiers, Electronics Letters, Vol. 30, pp. 2022-2023.

[2] Fabre, A. (1992). Gyrator implementation from commercially available transimpedance operational amplifiers, Electronics Letters, Vol. 28, pp. 263-264.

[3] Fabre, A. (1993). Insensitive voltage-mode and current-mode filters from commercially available transimpedance opamps, IEEE Proceedings, Part G, Vol. 140, pp. 319-321.

[4] Liu, S.-I. and Hwang, Y. S. (1994). Realisation of R-L and C-D impedances using a current feedback amplifier and its applications, Electronics Letters, Vol. 30, pp. 380381.

[5] Liu, S.-I. (1995). Universal filter using two current-feedback amplifiers, Electronics Letters, Vol. 31, pp. 629-630.

[6] Liu, S.-I. (1995). High input impedance filters with low component spread using current-feedback amplifiers, Electronics Letters, Vol. 31, pp. 1042-1043.

[7] Liu, S.-I. and Wu, D.-S. (1995). New current-feedback amplifier-based universal biquadratic filter, IEEE Transactions on Instrumentation and Measurement, Vol. 44, pp. 915-917. 

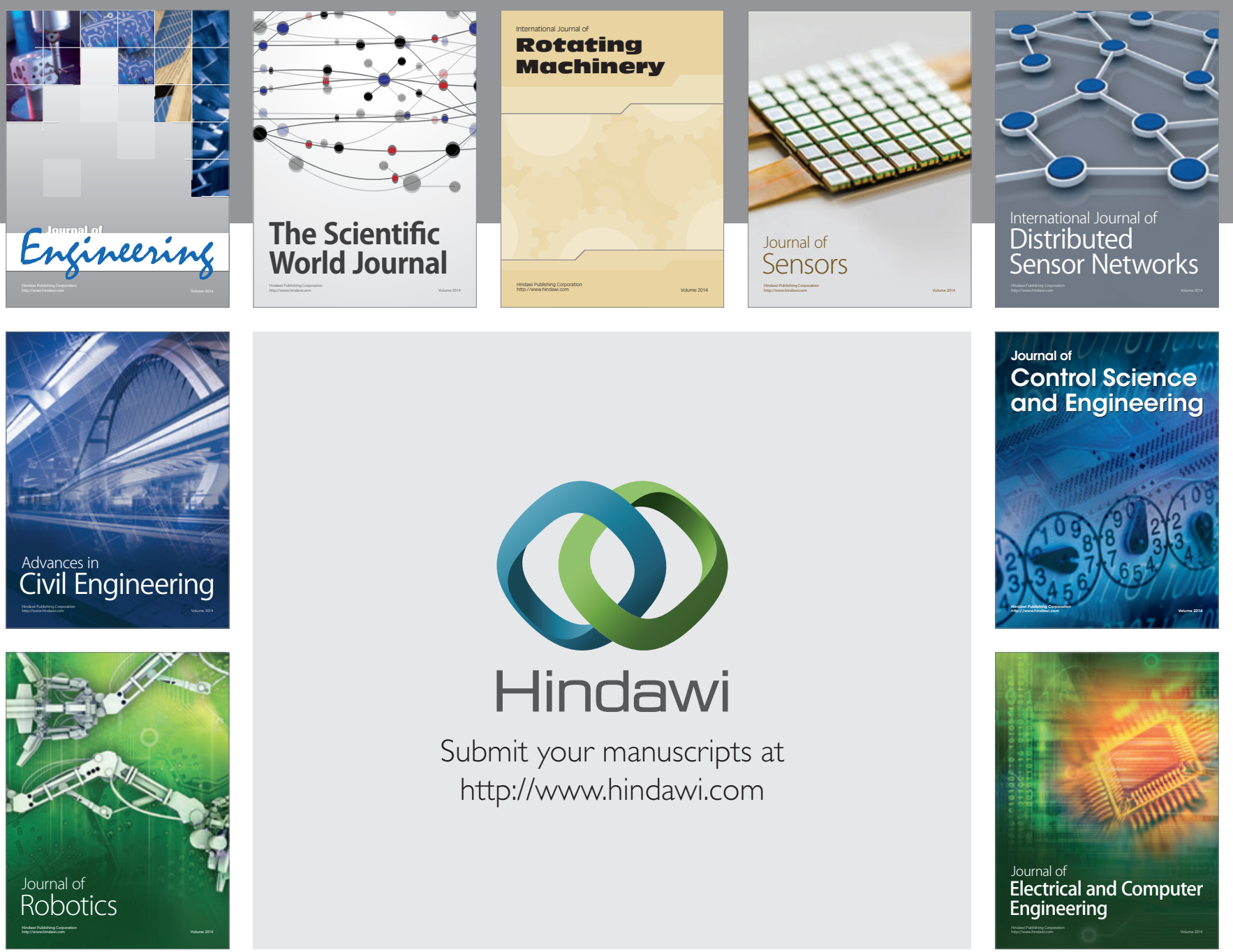

Submit your manuscripts at

http://www.hindawi.com
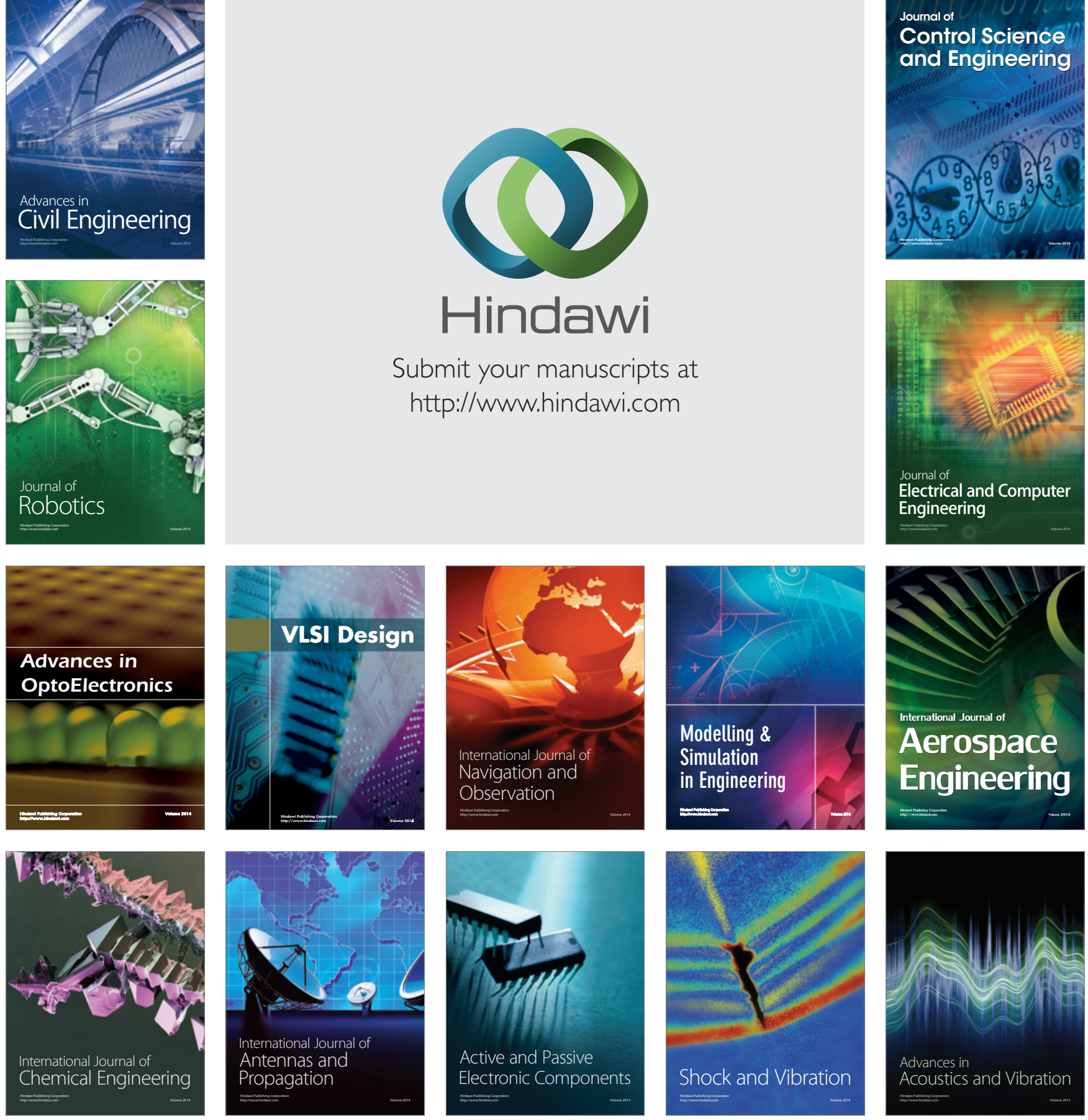